\title{
Patient perceptions of treatment and illness when prescribed multiple medicines for co-morbid type 2 diabetes
}

This article was published in the following Dove Press journal: Diabetes, Metabolic Syndrome and Obesity:Targets and Therapy 8 April 201I

Number of times this article has been viewed

\section{Rebecca J Stack' \\ Chris Bundy ${ }^{2}$ \\ Rachel A Elliott ${ }^{3}$ \\ John P New ${ }^{4}$ \\ J Martin Gibson ${ }^{4}$ \\ Peter R Noyce ${ }^{5}$}

'Sandwell and West Birmingham NHS Trust and University of Birmingham, Birmingham, UK; ${ }^{2}$ Manchester Academic Health Science Centre, University of Manchester, Manchester, UK; ${ }^{3}$ School of Pharmacy, University of Nottingham, Nottingham, UK; ${ }^{4}$ Salford Royal Foundation Trust, Salford, UK; ${ }^{5}$ School of Pharmacy and Pharmaceutical Sciences, University of Manchester, Manchester UK
Correspondence: Rebecca Stack School of Immunity and Infection, College of Medical and Dental Sciences, University of Birmingham, Birmingham, BI5 2TT, UK

Tel +44 I2। 4 I 46787

Fax +44 I2। 4I 46794

Email r.stack@bham.ac.uk
Abstract: Illness and treatment perceptions are vital for people self-managing co-morbid conditions with associated cardiovascular disease, such as type 2 diabetes (T2D). However, perceptions of a co-morbid condition and the use of multiple medicines have yet to be researched. This study investigated the illness and treatment perceptions of people with co-morbid T2D. The Brief Illness Perception Questionnaire (repeated for T2D, hypertension, and hyperlipidemia) and the Beliefs about Medicines Questionnaire Specific Concerns Scales (repeated for Oral hypoglycemic agents, anti-hypertensive medicines, and statins) were sent to 480 people managing co-morbid T2D. Data on the number of medicines prescribed were collected from medical records. Significantly different perceptions were found across the illnesses. The strongest effect was for personal control; the greatest control reported for T2D. Illness perceptions of T2D differed significantly from perceptions about hyperlipidemia. Furthermore, illness perceptions of T2D also differed from perceptions of hypertension with the exception of perceptions of illness severity. Hypertension and hyperlipidemia shared similar perceptions about comprehensibility, concerns, personal control, and timeline. Significant differences were found for beliefs about treatment necessity, but no difference was found for treatment concerns. When the number of medicines was taken as a between-subjects factor, only intentional non-adherence, treatment necessity beliefs, and perceptions of illness timeline were accounted for. Co-morbid illness and treatment perceptions are complex, often vary between illnesses, and can be influenced by the number of medicines prescribed. Further research should investigate relationships between co-morbid illness and treatment perception structures and self-management practices.

Keywords: co-morbidity, polypharmacy, illness perceptions, treatment perceptions, increasing numbers of medicines

\section{Introduction}

The Quality and Outcomes Framework for England tables 2008 to 2009 reported that the overall prevalence of diagnosed cases of diabetes was $5.1 \%(2,213,138$ people $) .{ }^{1}$ Seventy percent of people with type 2 diabetes (T2D) also have premature cardiovascular disease (CVD). ${ }^{2}$ The associated risk of CVD-linked mortality is higher for people with T2D. ${ }^{3-5}$ Co-morbid T2D, hypertension, and hyperlipidemia represent parts of the same overall pathophysiologic risk profile and would be treated using the same disease management plan. ${ }^{6}$

Optimal self-management is an integral part of medical management for co-morbid T2D and is needed to avoid long term microvascular and macrovascular complications. ${ }^{7}$ However, evidence suggests that the presence of co-morbidities negatively affects the ability of people to manage their conditions. ${ }^{8}$ For example, their motivation to act on risk 
factors (often requiring behavior change), ability to identifiy symptoms early, recognition of any deterioration of each condition, and successful management of complicated medication regimens may all be constrained. ${ }^{9}$ Furthermore, when multiple medicines are prescribed to people with co-morbid conditions, adherence can be adversely affected. ${ }^{10}$

Large, randomized controlled trials such as the United Kingdom Prospective Diabetes Study and the Collaborative Atorvastatin Diabetes Study advocate intensive pharmacological management of T2D. ${ }^{11,12}$ Most people with T2D are prescribed multiple medicines to improve metabolic control, lower serum glucose, cholesterol, and blood pressure. However, many studies have found that adherence is affected by the frequency of doses and the number of medicines prescribed. ${ }^{13}$ For example, Donnan et al found that once daily doses of oral hypoglycemic agents (OHAs) were associated with better adherence compared with multiple daily doses. ${ }^{14}$ Stack et al found that people managing co-morbid T2D often prioritized some medicines over others, with medicines for hyperlipidemia management being perceived as less important. ${ }^{15}$ Other studies found that when people are prescribed large numbers of medicines, adherence to medicines for hyperlipidemia management decreased. A review of antihypertensive medicines found that the adherence rate for a single daily dose was significantly higher than adherence to multiple daily doses. ${ }^{16}$ The way that multiple medicines are used may be driven by how patients understand and perceive their medicines, treatment, and co-morbid illness(es).

The self-regulation model provides a theoretical framework to explain how illness perceptions inform coping strategies including self-management behaviors. ${ }^{17,18}$ The self-regulation model describes the perceptions common to people managing an illness and how those with an illness such as T2D make sense of, cope with, adjust to, and manage their condition. Illness and treatment perceptions are linked to self-management behaviors such as adherence to medicines, ${ }^{19,20}$ lifestyle modification, and self-monitoring. ${ }^{21}$ The self-regulation model includes emotional (fear, worry, and depression) and cognitive (timeline, severity, identity, cause, and cure/control) representation of illness. Cognitive representations include: identity, that is, beliefs about the labels attached to an illness and the symptoms associated with that illness; beliefs about the cause, which can include lifestyle, environment, or genetic factors; timeline beliefs, which relate to the perceived duration of the illness and whether it is acute or time limited, chronic or cyclical; beliefs about consequences, which reflect a range of the potential costs of an illness; physical, psychological, social, or financial beliefs and, finally, cure or control beliefs related to perceived curability, perceived treatment control, and perceived individual control.

The self-regulation model has been extended to explore beliefs about medicines. Horne suggests that people hold general beliefs about medicines which refer to medicines as a category of treatment. ${ }^{22}$ General beliefs include perceptions about the general harm that medicines cause and perceptions about overuse. Specific beliefs about treatment cover necessity beliefs and concerns about medicines. Treatment necessity beliefs are perceptions about personal need for medication for both current and future health. Examples of treatment necessity beliefs include beliefs about the effectiveness of medicines or whether missing medications would have a negative impact on health. Concerns about medicines arise from beliefs about the potential negative effects of a medication. Little is known about the illness representations held by people living with co-existing or co-morbid conditions. Yet chronic medical conditions most often occur, not as a single impairment or risk factor, but as multiple or co-morbid conditions for which multiple medicines may have been prescribed. ${ }^{23}$

There is no research on the way people with $\mathrm{T} 2 \mathrm{D}$ perceive the presence of macrovascular complication as co-morbid conditions (particularly, hypertension and hyperlipidemia). The question of whether illness models of T2D differ (for example, more negative) from those for hypertension and hyperlipidemia needs to be addressed. Characterizing the personal models of patients is important, in order to develop care plans and facilitate self-management, because accurate understanding is pivotal to good care. Beliefs about co-morbid conditions may consist of separate perceptions of each individual illness (an additive model) or a more coherent understanding of how they interact (a multiplicative model).

This research aimed to characterize the illness perceptions of people managing the co-morbid conditions of T2D, hypertension, and hyperlipidemia.

\section{Method}

\section{Sample}

Eight hundred and seventy-eight people with T2D and CVD were identified from a secondary care diabetes database in the United Kingdom. This register incorporated prescribing information from secondary and primary care, which enhanced the reliability of the data used in this research. Patients aged 18 years or more were included if they were prescribed 1 or more oral hypoglycemic agents for the 
treatment of T2D, 1 or more anti-hypertensive drugs, and a statin for the treatment of hyperlipidemia. People with a care or nursing home address were excluded as they were assumed to have less personal control over taking their medicines than community dwelling populations. People who had not attended the diabetes clinic at the hospital where the study was undertaken within 12 months of the study starting were not approached. This technique improved the response rate and ensured that people were not sent mail from healthcare services with which they had little or no contact.

\section{Materials}

The Brief Illness Perceptions Questionnaire, IPQ(b), includes 1 item to represent each of the 7 main components of the IPQ-R: illness consequences; timeline; personal control; treatment control; identity; emotional concern; emotion and illness comprehensibility. ${ }^{24}$ Each item is measured on a 10-point Likert scale (from 1 to 10). The final, eighth, domain of the IPQ(b) asks respondents to state 3 causes of their illness. The IPQ(b) was repeated for T2D, hypertension, and hyperlipidemia separately to determine whether patient perceptions of each of these conditions differed.

The Beliefs about Medicines Specific Concerns Scales Questionnaire (BMQ) subscale measures treatment necessity beliefs and concerns about specific medication. ${ }^{25}$ Each item used a 5-point Likert scale; scores range between 1 and 5. The BMQ's Specific Concerns subscale assesses the perceived treatment necessity and perceived concerns about medicines. The BMQ scales for treatment necessity beliefs and treatment control were used to access perceived treatment necessity and concern about OHAs, anti-hypertensive medicines, and statins (each of the Specific Belief scales was presented 3 times).

\section{Number of medicines prescribed}

The number of medicines prescribed was extracted from a secondary care hospital's diabetes database. The number of medicines prescribed for T2D, hypertension, and hyperlipidemia were counted, added together, and used to construct a categorical between-subjects variable. To create a categorical variable, the number of medicines prescribed was divided into 3 groups. The groups were created to ensure an equal number of respondents for each category. Two hundred and thirty-two people were prescribed 3 medicines. This group was prescribed 1 medicine from each category: 1 OHA, 1 anti-hypertensive, and 1 statin. Those prescribed 3 medicines had the lowest number of medicines and were the only group to consistently have the same medicines. One hundred and two people were prescribed between 4 and 5 medicines; 146 people were prescribed 5 medicines or more; the maximum number of medicines prescribed in the group was 8 .

Participants were sent the entire questionnaire battery, and were asked to return it when complete. The questionnaire battery presented the BMQ first and then IPQ(b) for each condition. Half of the questionnaire batteries sent presented the T2D scales first, followed by the hypertension, and hyperlipidemia scales. The other half of the questionnaire batteries presented the hypertension, hyperlipidemia, and then the T2D scales.

\section{Procedure}

Research ethics approval was obtained. The collaborating diabetologist with consent to access patient medical records used the eligibility criteria to identify eligible adults from the diabetes register based on the medicines prescribed. Questionnaires, an invitation letter, and a free post return envelope were sent to eligible participants. Two reminders were sent to increase the response rate 2 and then 4 weeks after the initial mailing.

\section{Analysis}

A repeated-measures ANOVA was used to explore treatment variance in specific treatment beliefs across OHAs, anti-hypertensive medicines, and statins. A repeated measure ANOVA was used to explore variation in illness perceptions across T2D, hypertension, and hyperlipidemia. The number of medicines prescribed was added as a between-subjects factor to understand the effect of the number of medicines on treatment perceptions and illness perceptions.

\section{Results}

\section{Response rate}

Of the 878 questionnaires sent, 23 were returned as either 'not known at this address', or 'addressee has gone away', 42 people returned a blank questionnaire or declined participation directly, so that the 'active' sample included 855 people. Of those, 480 (56\%) questionnaires were finally returned.

\section{Sample characteristics}

Participants' mean age was 66.3 years (range: 28-94 years), with $64.6 \%$ male (data were missing in 3 cases), $91.7 \%$ white, $1.8 \%$ Asian, and $0.4 \%$ from other backgrounds. Data on ethnicity were missing for 25 cases. Table 1 shows the range of medicines prescribed to participants. Of participants, $21.5 \%$ reported an annual household income of less than $£ 19,999 ; 7.9 \%$ reported an annual income above $£ 20,000$; 
Table I Numbers of participants prescribed each medication

\begin{tabular}{lll}
\hline $\begin{array}{l}\text { Category of } \\
\text { medication }\end{array}$ & $\begin{array}{l}\text { Name of medication } \\
\text { sub-category }\end{array}$ & $\begin{array}{l}\text { Number (\%) } \\
\text { prescribed }\end{array}$ \\
\hline OHAs & Metformin & $388(80.33 \%)$ \\
& Glitazones & $74(15.42 \%)$ \\
& Sulfonylureas & $0(0 \%)$ \\
Anti- & Angiotensin I converting enzyme & $324(67.5 \%)$ \\
hypertensives & inhibitors & \\
& Calcium channel blockers & $211(43.96 \%)$ \\
& Angiotensin II receptor antagonists & $128(26.67 \%)$ \\
& Diuretics & $126(26.25 \%)$ \\
& Alpha-blocker & $49(10.21 \%)$ \\
HMG CoA reductase inhibitor (statins) & $0(0 \%)$ \\
\hline
\end{tabular}

$27.7 \%$ reported that they were retried, and $42.9 \%$ choose not to disclose their annual household income.

\section{Within-subjects effects}

Within-subjects ANOVAs were used to compare the mean scores given on $8 \mathrm{IPQ}(\mathrm{b})$ domains/items in relation to T2D, hypertension, and hyperlipidemia, and the BMQ subscales in relation to OHAs, anti-hypertensive medicines, and statins. Table 2 shows the Wilks lambda statistic for each within-subjects ANOVA. Differences between perceptions held about T2D, hypertension, and hyperlipidemia were significant and partial eta squared was used to interpret the magnitude of the difference. The largest effect was reported for personal control indicating a large difference in perceived personal control between the illnesses.

\section{Illness severity}

Participants perceived the severity for T2D to be greater than hyperlipidemia (mean difference $=1.285, P<0.001$, confidence interval [CI] [95\%] 0.624-1.947) but not hypertension (mean difference $=0.634, P=0.279, \mathrm{CI}[95 \%]-0.037$ to 1.305). Hypertension was perceived as more severe than hyperlipidemia (mean difference $=0.652, P<0.001$, CI [95\%] 0.384-0.919).

\section{Illness timeline}

T2D was thought to be longer lasting than hypertension (mean difference $=1.201, P<0.001$, CI [95\%] 0.921-1.481) and hyperlipidemia (mean difference $=1.434, P<0.001$, CI [95\%] 1.139-1.728). Timeline perceptions of hypertension and hyperlipidemia were also significantly different (mean difference $=0.233, P<0.001$, CI [95\%] -0.034 to $0.5)$; timeline perceptions of hypertension were thought to be longer.

\section{Personal control}

Participants perceived greater personal control over T2D than either hypertension (mean difference $=0.501, P<0.001, \mathrm{CI}$ [95\%] 0.203-0.8) or hyperlipidemia (mean difference $=0.46$, $P<0.001$ CI [95\%] 0.157-0.763). Personal control perceptions for hypertension and hyperlipidemia were not significantly different (mean difference $=0.129, P=1.000$, CI [95\%] -0.351 to 0.269$)$.

\section{Treatment control}

Participants believed their treatment was more likely to control T2D than the subsequent treatment for either hypertension (mean difference $=0.475, P<0.001$ CI [95\%] $0.259-0.69$ ) or hyperlipidemia (mean difference $=0.754$, $P<0.001$, CI [95\%] 0.522-0.986). The difference between perceptions of treatment control for hypertension and hyperlipidemia was also significant (mean difference $=0.279$, $P<0.05$, CI [95\%] 0.026-0.532); participants held significantly stronger treatment control perceptions for hypertension.

\section{Illness identity}

More symptoms were reported for T2D than either hypertension (mean difference $=0.68, P<0.001$, CI [95\%] $0.358-1.001$ ) or hyperlipidemia (mean difference $=1.245$, $P<0.001$, CI [95\%] 0.926-0.1.565). Similarly, significantly more symptoms were perceived for hypertension

Table 2 The within-subjects ANOVA statistics for IPQ(b) items

\begin{tabular}{lllll}
\hline & $\begin{array}{l}\text { Wilks } \\
\text { lambda }\end{array}$ & F & df & Partial eta \\
squared
\end{tabular}


than hyperlipidemia (mean difference $=0.566, P<0.001$, CI [95\%]) 0.299-0.833).

\section{Illness concerns}

More concerns were reported for T2D than for either hypertension (mean difference $=0.426, P<0.05$, CI $[95 \%]$ $0.075-0.778$ ) or hyperlipidemia (mean difference $=0.686$, $P<0.001$, CI [95\%] 0.332-1.04). No significant difference was found between the number of concerns for hypertension and hyperlipidemia (mean difference $=0.259, P=0.195, \mathrm{CI}$ [95\%] -0.078 to 0.596$)$.

\section{Illness comprehensibility}

Greater understanding was reported for T2D than either hypertension (mean difference $=0.799, P<0.001$, CI [95\%] $0.475-1.124$ ) or hyperlipidemia (mean difference $=0.943$, $P<0.001$, CI [95\%] 0.613-1.274) but not between hypertension and hyperlipidemia (mean difference $=0.144, P=0.683$, CI [95\%] -0.143 to 0.431$)$.

\section{Illness emotion}

The level of emotional distress associated with T2D was significantly higher than either hypertension (mean difference $=0.438, P<0.001$, CI [95\%] 0.147-0.73) or hyperlipidemia (mean difference $=1.192, P<0.001$, CI [95\%] 0.884-1.501). Also, emotional distress associated with hypertension was greater than that associated with hyperlipidemia (mean difference $=0.754, P<0.001$, CI [95\%] 0.496-1.012).

\section{Treatment necessity}

A repeated-measures ANOVA found a significant difference across medicines for treatment necessity beliefs (Wilks lambda $=0.804, \mathrm{~F}=47.217, d f=2,388, P<0.000$, partial eta squared $=0.334$ ). A post hoc analysis revealed that $\mathrm{OHAs}$ were associated with greater treatment necessity beliefs compared with anti-hypertensives (mean difference $=1.153, P<0.000$, CI [95\%] 0.713-1.593) and statins (mean difference $=2.694$, $P<0.000$, CI [95\%] 2.219-3.169). Anti-hypertensives had significantly higher treatment necessity beliefs compared with treatment necessity beliefs for statins (mean difference $=1.542$, $P<0.000$, CI [95\%] 1.079-2.004).

\section{Treatment concerns}

No significant difference was found for treatment concerns across medicines (Wilks lambda $=1, \mathrm{f}=0.068, d f=2,372$, $P=0.935)$. Therefore, concerns about medicines did not vary between OHAs, anti-hypertensives, and statins.

\section{Influence of the number of medicines prescribed on perceptions}

The between-subjects effect for treatment necessity beliefs was not significant ( $\mathrm{f}=2.502, d f=2,370, P=0.083$ ). Therefore, treatment necessity beliefs did not increase as the number of medicines prescribed increased. The interaction between treatment necessity beliefs and the number of medicines prescribed was significant $(\mathrm{f}=2.559, d f=4,370$, $P<0.05$, partial eta squared $=0.014)$. The interaction was interpreted using a line graph. Figure 1 shows that treatment necessity beliefs for OHAs, anti-hypertensives, and statins have a different relationship with the number of medicines. Treatment necessity beliefs for anti-hypertensives differ for low, medium, and high numbers of medicines, whereas treatment necessity beliefs for statins and OHAs do not appear to be affected by the number of medicines prescribed.

The between-subjects effect for treatment concerns was not significant ( $\mathrm{f}=0.220, d f=2,370, P=0.802$ ): therefore, the number of medicines prescribed did not influence the level of concern reported. Treatment concerns did not differ across OHA, anti-hypertensives, and statins, and the interaction between treatment concerns and the number of medicines prescribed was not significant $(\mathrm{f}=1.696, d f=4,370$, $P=0.150)$.

The between-subjects effect for illness severity was significant $(\mathrm{f}=5.003, d f=2,314, P<0.01$, partial eta squared $=0.031)$. Therefore, scores on the IPQ(b) illness severity item increased as the number of medicines prescribed increased. The interaction was not significant: therefore, the number of medicines prescribed did not affect the illness severity scores given for individual illnesses ( $\mathrm{f}=0.974 d f=2,371$, $P=0.390$ ). There was a consistent relationship between the number of medicines and illness severity across illnesses.

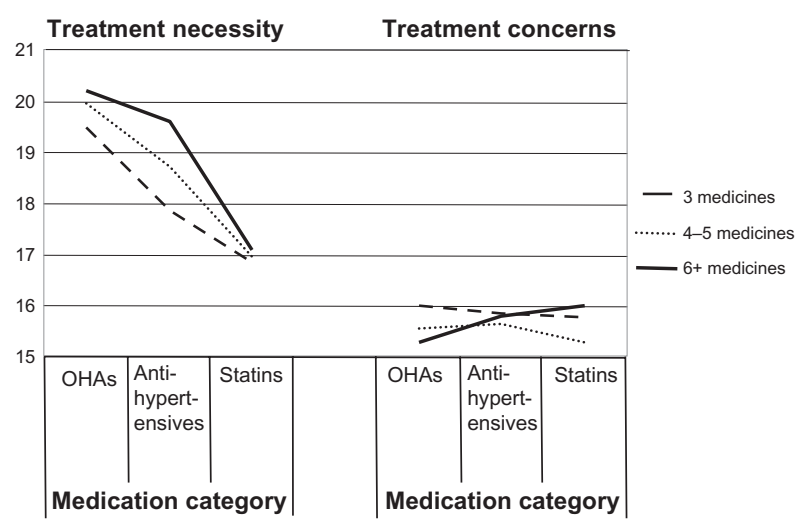

Figure I Treatment necessity beliefs and treatment concerns across medicines for low, medium and high numbers of medicines. Abbreviation: OHAs, oral hypoglycemic agents. 
The between-subjects effect for illness timeline was not significant: therefore, IPQ(b) illness timeline scores did not significantly increase as the number of medicines prescribed increased ( $\mathrm{f}=2.456, d f=2,375, P=0.087$ ). The interaction between timeline scores across illnesses and the number of medicines prescribed was significant. Thus increases in the number of medicines prescribed affect timeline scores for the illnesses investigated in different ways ( $\mathrm{f}=4.132, d f=2,371, P<0.01$, partial eta squared $=0.026)$. Hyperlipidemia was associated with greater timeline perceptions (perceived to last for longer) for people prescribed 6 or more medicines.

The between-subjects effect for personal control was not significant: perceptions of personal control did not differ significantly as more medicines were prescribed $(\mathrm{f}=0.171$, $d f=2,314, P=0.843)$. The interaction between the number of medicines prescribed and personal control beliefs across illnesses was not significant ( $\mathrm{f}=1.233, d f=2,371, P=0.296$ ). Therefore, personal control did not significantly increase as more medicines were prescribed and this relationship was consistent across illnesses.

The between-subjects effect for treatment control was not significant: treatment control did not differ as more medicines were prescribed ( $\mathrm{f}=0.716, d f=2,391, P=0.489$ ). The interaction was not significant: therefore, the number of medicines did not affect perceptions of treatment control for individual illnesses ( $\mathrm{f}=1.519, d f=2,371, P=0.198$ ).

The between-subjects effect for identity was significant $(\mathrm{f}=3.610, d f=2,314, P<0.05$, partial eta squared $=0.022)$ : therefore, identity perceptions (ie, the level of symptoms reported) were affected by the number of medicines prescribed. For example, more symptoms were reported for higher numbers of medicines. The interaction between identity beliefs and the number of medicines prescribed was also significant $(\mathrm{f}=2.522, d f=2,371, P<0.05$, partial eta squared $=0.043$ ). Therefore, the number of medicines prescribed appears to affect identity beliefs about different illnesses in different ways. Increasing identity beliefs for $\mathrm{T} 2 \mathrm{D}$ and hypertension are related to increasing numbers of medicines. Identity beliefs for hyperlipidemia do not increase as more medicines are prescribed.

The between-subjects effect for illness concerns was not significant: therefore, illness concerns did not significantly differ (or increase) as more medicines were prescribed ( $\mathrm{f}=0.246, d f=2,314, P=0.782$ ). The number of medicines prescribed did not influence illness concerns about individual illnesses ( $\mathrm{f}=2.109, d f=2,371, P=0.079$ ). Therefore, the interaction was not significant.
The between-subjects analysis did not find a significant effect between the number of medicines prescribed and illness emotion ( $\mathrm{f}=0.915, d f=2,314, P=0.173$ ). The interaction was not significant: therefore, the number of medicines prescribed did not affect emotions for individual illnesses ( $f=1.539$, $d f=2,371, P=0.193$ ). The between-subjects analysis found that illness comprehensibility did not differ as more medicines were prescribed $(\mathrm{f}=0.711, d f=2,314, P=0.711)$. The interaction was not significant and the number of medicines prescribed did not affect illness comprehensibility levels for different illnesses ( $\mathrm{f}=1.858, d f=2,371, P=0.118)$.

\section{Conclusion}

Differences in illness perceptions were found between T2D, hypertension, and hyperlipidemia. The strongest differences found were for personal control. Participants perceived that they had greater personal control over T2D than other conditions. This suggests that they or healthcare professionals find T2D easier to control than other conditions. This finding may reflect the greater focus on diabetes structured education programmes for T2D self-management. Structured education sessions may empower patients to feel as though they have greater control over T2D, and that the treatment offered also provides better control, but to neglect CVD self-management. Other studies have reported that patients receiving structured education have a lack of awareness of macrovascular disease. ${ }^{26}$ Furthermore, participants in this study also reported a greater understanding of T2D compared with other conditions, supporting the conclusion that a greater awareness of T2D exists, whereas CVD is generally less well understood and controlled. This may have implications for the way each condition is self-managed. For example, the consequences for self-management may include people focusing on reducing blood sugars rather than cholesterol reduction. How far patients' beliefs about these aspects of their conditions affect their self-management is not clear from this study and warrants further work. Also, other ways of testing the level of factual understanding of co-morbid conditions was not examined within this study and variations in healthcare literacy among participants may limit these findings.

T2D and hyperlipidemia were found to be significantly different for all IPQ(b) items. Therefore, participants appeared to perceive T2D and hyperlipidemia as very different conditions. This finding questions whether patients perceive T2D and hyperlipidemia to be part of a co-morbid condition or as separate illnesses with individual etiologies and management strategies. If a separation had been 
perceived between these two conditions, we could conclude that the level of healthcare literacy among people with comorbid conditions is low, especially knowledge of how elements of co-morbid T2D are related to each other. Similarly, perceptions of hypertension were also significantly different to all perceptions of T2D, with the exception of perceptions of illness severity; the consequences of T2D and hypertension were perceived to have a similar level of severity. Overall hyperlipidemia was perceived as a less severe condition with less severe consequences.

Perceptions of timeline, emotions, and identity showed a similar pattern of differences, with T2D being perceived to last longer, cause more emotional distress and a greater number of symptoms than hypertension and hyperlipidemia. Furthermore, hypertension was perceived to last longer, cause more emotional distress, and a greater number of symptoms than hyperlipidemia. T2D caused a greater amount of emotional distress than other conditions. Despite T2D, hypertension and hyperlipidemia being conditions for which symptoms are mild and in some cases asymptomatic, more symptoms were reported for T2D. The greater reporting of symptoms may be related to the higher level of distress associated with T2D. Other research has found a link between depression and the reporting of symptoms in $\mathrm{T} 2 \mathrm{D}$; further links were made to self-management with lower levels of adherence being reported, ${ }^{27}$ but more research is required to identify the relationship between emotion, symptoms, and self-management in co-morbid T2D.

Necessity beliefs did not change as more medicines were prescribed; however, the interaction between treatment necessity beliefs and the number of medicines prescribed was significant. Therefore, the number of medicines influences treatment necessity beliefs for a specific medication, possibly anti-hypertensives. The number of medicines prescribed did not influence treatment concerns and the interaction was not significant. Therefore, concerns about medicines for people with co-morbid T2D were stable and consistent across conditions and between groups. This confirms the suggestion that concerns about treatment may not need to be differentiated (ie, each type of concern addressed) in an intervention. This finding suggests that a similar approach to addressing treatment concerns can be taken for those with varying numbers of medicines.

Perceptions of treatment control did not significantly differ as more medicines were prescribed, and the interaction was not significant. Also, perceptions of illness severity, illness personal control, illness concerns, illness emotional concerns, and illness comprehensibility did not significantly differ as more medicines were prescribed, and the interaction was not significant. Intuitively more medicines should have been associated with more concerns and emotional distress. Yet our results indicate that more medicines are not related to concerns or emotional distress, which may be explained by the quality of care received from health providers by those prescribed large numbers of medicines. Other studies indicate that as multi-morbid and co-morbid conditions increase, the quality of care patients receive also increases. ${ }^{28-30}$ This suggests that people managing more severe conditions, and possibly more medicines, may experience less distress and cope better because of increases in the quality of care they receive. Further research is needed to explore the impact of quality of care on the concerns and emotional distress associated with greater numbers of medicines.

Perceptions of illness timeline were not affected by the number of medicines prescribed. The interaction was significant, however, and illness timeline perceptions for hypertension had a significantly different interaction with the number of medicines prescribed, compared with timeline perceptions for T2D and hyperlipidemia. Therefore, perceptions of hypertension timeline and treatment necessity beliefs for anti-hypertensives are both higher for those who are prescribed larger numbers of medicines. Identity perceptions (the level of symptoms reported) were affected by the number of medicines prescribed and the interaction was significant. Increased reporting of symptoms for T2D and hypertension were related to increasing numbers of medicines, while identity beliefs for hyperlipidemia did not increase as more medicines were prescribed.

This study was the first to examine differences and cohesiveness in co-morbid illness and treatment perceptions. We have shown that people with co-morbid T2D have multiple beliefs about their condition, which do not reflect 1 coherent illness. Our findings indicate that the IPQ(b), BMQ specific concerns, and BMQ necessity subscale when repeated for each medicine and illness can detect differences among beliefs between conditions. Also, this research is the first to examine the role that the number of medicines prescribed has on illness and treatment perceptions. We acknowledge that the IPQ(b) and BMQ were not designed to detect withingroup differences but their use in this way has extended their possible utility. This draws attention to the limitations of the self-regulation model when applied to the study of co-morbid conditions. Further research on the primacy of beliefs in co-morbid conditions, and measures that reflect this complexity, is required. 


\section{Limitations}

This study has limitations, although a good response rate for an unsolicited survey was achieved. There is still a risk that the survey may have over sampled people with particular self-management behaviors or perceptions who present for secondary care. Furthermore, this cross-sectional study gives us little information about how co-morbid illness perceptions develop over time. The level of factual understanding of co-morbid conditions was not examined within this study and variations in healthcare literacy among participants may limit these findings. Future research should address this issue as it may account for some of the variance in illness beliefs. Co-morbid T2D, hypertension, and hyperlipidemia are related conditions and the lifestyle changes that are required overlap. Future research should explore perceptions of other co-morbidities that require explicitly different sets of management activities, and investigate the differences in illness and treatment perceptions.

\section{Relevance to clinical practice}

Individuals self-managing co-morbid T2D are likely to hold different perceptions of their illness; this variation has been linked to the number of medicines prescribed, and therefore, variations in self-management behaviors are also likely. For example, the approach taken to manage T2D and hyperlipidemia may differ because the beliefs held for both conditions are significantly different. Qualitative research has shown that patients often view T2D as severe and requiring pharmacological management, while hyperlipidemia is perceived to be less severe, with many believing that this condition should be managed through lifestyle modification and that cholesterol lowering medicines were unnecessary. ${ }^{15}$ Healthcare professionals must recognize that people self-managing co-morbid conditions and prescribed multiple medicines, are likely to hold different perceptions about their conditions which may be influenced by the number of medicines they are being prescribed, and may adopt different self-management strategies in response to their beliefs.

Understanding patients' personal models of co-morbid conditions is essential for understanding self-management behaviors in people with T2D. Interventions that reduce risk factors through lifestyle modification, could improve health outcomes for people with T2D, particularly if they target unhelpful or incorrect perceptions. ${ }^{31}$ The findings of this research indicate that exploring perceptions about co-morbid conditions and multiple medicines is not a straightforward task for healthcare professionals and more valid and reliable measures are required to address this complexity.

\section{Disclosure}

The authors have no conflict of interest to disclose.

\section{References}

1. QoF. Quality and Outcomes Framework (QOF) for April 2008-March 2009, England. http://www.ic.nhs.uk/statistics-and-data-collections/ supporting-information/audits-and-performance/the-quality-andoutcomes-framework/qof-2008/09/data-tables/prevalence-data-tables. Accessed July 22, 2010

2. Laakso M. Cardiovascular disease in type 2 diabetes: challenge for treatment and prevention. J Intern Med. 2001;249(3):225-235.

3. Booth GL, Kapral MK, Fung, Tu JV. Recent trends in cardiovascular complications among men and women with and without diabetes. Diabetes Care. 2006;29(1):32-37.

4. Booth GL, Kapral MK, Fung K, Tu JV. Relation between age and cardiovascular disease in men and women with diabetes compared with non-diabetic people: a population-based retrospective cohort study. Lancet. 2006;368(9529):29-36.

5. Haffner SM, Lehto S, Ronnemaa T, Pyorala K, Laakso M. Mortality from coronary heart disease in subjects with type 2 diabetes and in nondiabetic subjects with and without prior myocardial infarction. N Engl J Med. 1998;339(4):229-234.

6. Piette JD, Kerr EA. The impact of comorbid chronic conditions on diabetes care. Diabetes Care. 2006;29(3):725-731.

7. Jarvis J, Skinner TC, Carey ME, Davies MJ. How can structured selfmanagement patient education improve outcomes in people with type 2 diabetes? Diabetes Obes Metab. 2010;12(1):12-19.

8. Morrow AS, Haidet P, Skinner J, Naik AD. Integrating diabetes self-management with the health goals of older adults: a qualitative exploration. Patient Educ Couns. 2008;72(3):418-423.

9. Jowsey T, Jeon YH, Dugdale P, Glasgow NJ, Kljakovic M, Usherwood T. Challenges for co-morbid chronic illness care and policy in Australia: a qualitative study. Aust New Zealand Health Policy. 2009;6:22.

10. Stack RJ, Bundy C, Elliott RA, Gibson JM, New JP, Noyce PR. Intentional and unintentional non-adherence in community dwelling people with type 2 diabetes: the effect of varying numbers of medicines. Journal of Diabetes and Vascular Disease. 2010;10(3):148-152.

11. Bretzel RG, Voigt K, Schatz H. The United Kingdom Prospective Diabetes Study (UKPDS) implications for the pharmacotherapy of type 2 diabetes mellitus. Exp Clin Endocrinol Diabetes. 1998;106(5): 369-372.

12. Colhoun HM, Betteridge DJ, Durrington PN, et al; CARDS investigators. Primary prevention of cardiovascular disease with atorvastatin in type 2 diabetes in the Collaborative Atorvastatin Diabetes Study (CARDS): multicentre randomised placebo-controlled trial. Lancet. 2004;364(9435):685-696.

13. Paes AH, Bakker A, Soe-Agnie CJ. Impact of dosage frequency on patient compliance. Diabetes Care. 1997;20(10):1512-1517.

14. Donnan PT, MacDonald TM, Morris AD. Adherence to prescribed oral hypoglycaemic medication in a population of patients with Type 2 diabetes: a retrospective cohort study. Diabet Med. 2002;19(7): 279-284.

15. Stack RJ, Elliott RA, Noyce PR, Bundy C. A qualitative exploration of multiple medicines beliefs in co-morbid diabetes and cardiovascular disease. Diabet Med. 2008;25(10):1204-1210.

16. Iskedjian M, Einarson TR, MacKeigan LD, et al. Relationship between daily dose frequency and adherence to antihypertensive pharmacotherapy: evidence from a meta-analysis. Clin Ther. 2002; 24(2):302-316.

17. Leventhal $\mathrm{H}$, Benyamini $\mathrm{Y}$, Brownlee $\mathrm{S}$, et al. Illness representations: theoretical foundations. In: Petrie KP, Weinman JA, editors. Perceptions of Health and Illness. Amsterdam: Harwood Academic Publishers; 1997:19-46.

18. Leventhal H, Nerenz DR, Steele DJ. Illness representations and coping with health threats. In: Taylor SE, Singer JE, editors. Handbook of Psychology and Health. Hillsdale, NJ. Lawrence Erlbaum; 1984:219-252. 
19. Horne R, Weinman J. Patients' beliefs about prescribed medicines and their role in adherence to treatment in chronic physical illness. J Psychosom Res. 1999;47(6):555-567.

20. Leventhal $\mathrm{H}$. The role of theory in the study of adherence to treatment and doctor-patient interactions. Med Care. 1985;23(5):556-563.

21. French DP, Wade AN, Yudkin P, Neil HA, Kinmonth AL, Farmer AJ. Self-monitoring of blood glucose changed non-insulin-treated Type 2 diabetes patients' beliefs about diabetes and self-monitoring in a randomized trial. Diabet Med. 2008;25(10):1218-1228.

22. Horne R. Patients' beliefs about treatment: the hidden determinant of treatment outcome? J Psychosom Res. 1999;47(6):491-495.

23. Fortin M, Soubhi H, Hudon C, Bayliss EA, van der Akker M. Multimorbidity's many challenges. BMJ. 2007;334(7602):1016-1017.

24. Broadbent E, Petrie KJ, Main J, Weinman J. The brief illness perception questionnaire. J Psychosom Res. 2006;60(3):631-637.

25. Horne R, Weinman J, Hankins M. The Beliefs about Medicines Questionnaire: The development and evaluation of a new method for assessing the cognitive representation of medication. Psychol Health. 1999;14(1):1-24.
26. Smith SM, O'Leary M, Bury G, et al. A qualitative investigation of the views and health beliefs of patients with Type 2 diabetes following the introduction of a diabetes shared care service. Diabet Med. 2003; 20(10):853-857.

27. Ciechanowski PS, Katon WJ, Russo JE, Hirsch IB. The relationship of depressive symptoms to symptom reporting, self-care and glucose control in diabetes. Genl Hosp Psychiatry. 2003;25(4):246-252.

28. Min LC, Wenger NS, Fung C, et al. Multimorbidity is associated with better quality of care among vulnerable elders. Med Care. 2007;45(6): $480-488$.

29. Higashi T, Wenger NS, Adams JL, et al. Relationship between number of medical conditions and quality of care. N Engl J Med. 2007;356(24): 2496-2504.

30. Hudon C, Fortin M, Dubois MF, Almirall J. Comorbidity and glycemia control among patients with type 2 diabetes in primary care. Diabetes Metab Syndr Obes. 2008;1:33-37

31. Fisher EB, Brownson CA, O'Toole ML, et al. The Robert Wood Johnson Foundation Diabetes Initiative: demonstration projects emphasizing self-management. Diabetes Educator. 2007;33(1):83-94.

\section{Publish your work in this journal}

Diabetes, Metabolic Syndrome and Obesity: Targets and Therapy is an international, peer-reviewed open-access journal committed to the rapid publication of the latest laboratory and clinical findings in the fields of diabetes, metabolic syndrome and obesity research. Original research, review, case reports, hypothesis formation, expert opinion and commentaries are all considered for publication. The manuscript management system is completely online and includes a very quick and fair peer-review system, which is all easy to use. Visit http://www.dovepress.com/testimonials.php to read real quotes from published authors. 